\title{
BMJ Open Cohort profile: Development and profile of a population-based, retrospective cohort of diagnosed people living with HIV in Ontario, Canada (Ontario HIV Laboratory Cohort)
}

To cite: Liu J, Wilton J, Sullivan A, et al. Cohort profile: Development and profile of a population-based, retrospective cohort of diagnosed people living with HIV in Ontario, Canada (Ontario HIV Laboratory Cohort). BMJ Open 2019:9:e027325. doi:10.1136/ bmjopen-2018-027325

- Prepublication history and additional material for this paper are available online. To view these files, please visit the journal online (http://dx.doi. org/10.1136/bmjopen-2018027325).

Received 16 0ctober 2018 Revised 8 February 2019 Accepted 12 March 2019

Check for updates

(C) Author(s) (or their employer(s)) 2019. Re-use permitted under CC BY-NC. No commercial re-use. See rights and permissions. Published by BMJ.

For numbered affiliations see end of article.

Correspondence to

Dr Abigail E Kroch;

akroch@ohtn.on.ca

\section{ABSTRACT}

Purpose Population-based cohorts of diagnosed people living with HIV (PLWH) are limited worldwide. In Ontario, linked HIV diagnostic and viral load (VL) test databases are centralised and contain laboratory data commonly used to measure engagement in HIV care. We used these linked databases to create a population-based, retrospective cohort of diagnosed PLWH in Ontario, Canada.

Participants A datamart was created by integrating diagnostic and $V L$ databases and linking records at the individual level. These databases contain information on laboratory test results and sociodemographic/clinical information collected on requisition/surveillance forms. Datamart individuals enter our cohort with the first record of a nominal HIV-positive diagnostic test (1985-2015) or VL test (1996-2015), and remain unless administratively lost to follow-up (LTFU; no VL test for $>2$ years and no VL test in later years). Non-nominal diagnostic tests are excluded as they lack identifying information to permit linkage to other tests. However, individuals diagnosed non-nominally are included in the cohort with record of a VL test. The LTFU rule is applied to indirectly censor for death/out-migration. Findings to date As of the end of 2015, the datamart contained 40372 HIV-positive diagnostic tests and 23851 individuals with $\geq 1 \mathrm{VL}$ test. Almost half $(46.3 \%)$ of the diagnostic tests were non-nominal and excluded, although this was lower $(\sim 15 \%)$ in recent years. Overall, 29587 individuals have entered the cohort-contributing 229302 person-years of follow-up since 1996. Between 2000 and 2015, the number of diagnosed PLWH (cohort individuals not LTFU) increased from 8859 to 16110 , and the percent who were aged $\geq 45$ years increased from $29.1 \%$ to $62.6 \%$. The percent of diagnosed PLWH who were virally suppressed $(<200$ copies $/ \mathrm{mL}$ ) increased from $40.7 \%$ in 2000 to $79.5 \%$ in 2015.

Future plans We plan to conduct further analyses of HIV care engagement and link to administrative databases with information on death, migration, physician billing claims and prescriptions. Linkage to other data sources will address cohort limitations and expand research opportunities.

\section{Strengths and limitations of this study}

- Our cohort is population-based and contains clinical laboratory data commonly used to measure the HIV cascade. Ontario is a relatively large jurisdiction ( 14 million people) and, as of the end of 2015, our cohort included data on almost 30000 diagnosed individuals.

- To the best of our knowledge, our study is one of only a few published population-based cohorts of diagnosed people living with HIV with individual-level linkage from diagnosis to viral suppression, despite such data sources being recommended as optimal for HIV cascade measurement over time.

- In the absence of death and out-migration data, we applied an administrative lost to follow-up (LTFU) rule (>2 years with no viral load [VL] test, and no VL test in later years) which may have led to the inappropriate censorship of some individuals. (However, individuals assessed as LTFU are not permanently censored and can re-enter the cohort with a subsequent record of a VL test.)

- Our cohort may be biased towards not adequately including/retaining individuals who never link to care after diagnosis, but the number of such individuals may be small.

- Certain sociodemographic and clinical information collected on requisition/surveillance forms are missing for a large proportion of participants, including race/ethnicity (84\%), HIV exposure category (49\%), most recent CD4 count (43\%) and use of antiretroviral treatment medications $(20 \%)$.

\section{INTRODUCTION}

Despite advances in HIV treatment and prevention, people living with HIV (PLWH) continue to experience worse health outcomes compared with those who are HIV-negative, ${ }^{1-4}$ and rates of new HIV 
diagnoses have not decreased substantially in many high-income countries. ${ }^{5-7}$ These realities are in contrast to the health and HIV transmission outcomes achieved under optimal care and treatment conditions. Indeed, research shows that early achievement of viral load (VL) suppression through antiretroviral treatment (ART) can lead to a life expectancy approaching that of HIV-negative individuals ${ }^{8-10}$ and can also eliminate the risk of transmitting HIV to sex partners. ${ }^{11} 12$ Gaps in HIV care and treatment, ${ }^{13}$ among other factors, ${ }^{14-16}$ are contributing to the higher morbidity/mortality experienced by PLWH as well as the ongoing transmission of HIV at the population level. ${ }^{13} 17$

In recent years, the HIV cascade has become a commonly used framework for measuring gaps in HIV care and organising the response to HIV. ${ }^{13}$ The cascade refers to the continuum of steps through which PLWH progress in order to achieve viral suppression. These steps include testing and diagnosis, linkage to and retention in HIV medical care and initiation of and adherence to ART. Measuring the HIV care cascade in different jurisdictions is now a priority and central to several HIV/AIDS strategies, including the UNAIDS' 90-90-90 strategy ${ }^{18}$ and Ontario's provincial HIV/AIDS Strategy to $2026 .^{19}$

Population-based cohorts of diagnosed PLWH are considered optimal for measurement of the HIV cascade over time, ${ }^{20}{ }^{21}$ but a 2015 systematic review identified only one jurisdiction (the Canadian province of British Columbia) ${ }^{22}{ }^{23}$ using such a data source for cascade measurement. ${ }^{20}$ Instead, many cascade studies draw on relatively small clinical cohorts of PLWH who are already in care, which are unlikely to be representative of all PLWH in a jurisdiction and cannot be used to construct a full cascade. Consequently, there has been a call for '[i] ncreased investments in population-based cohorts ... to complement clinical cohorts for ... longitudinal cascade analyses'. ${ }^{21}$

Ontario is the largest of the 13 provinces/territories in Canada, with a population of almost 14 million people in $2015^{24}$ and the highest number of HIV diagnoses in the country. About $40 \%$ of the more than 80000 HIV cases in Canada between 1985 and 2016 were diagnosed in Ontario. ${ }^{725}$ Similar to other high-income countries,${ }^{56}$ the rate of new HIV diagnoses in Ontario is not decreasing ${ }^{26}$; mortality is higher among PLWH compared with the general population, ${ }^{1}$ and current understanding of the HIV cascade is largely derived from surveys and clinical cohorts that are not population based. ${ }^{27} 28$ New data sources are needed to inform HIV policy and programming in Ontario.

Large clinical and administrative databases contain information that is routinely collected during encounters with the healthcare system and are increasingly being used in research. ${ }^{29-32}$ These databases can provide access to large populations and are generally more representative (ie, population based) and less expensive/time intensive than common study designs such as surveys and prospective cohort studies. ${ }^{29} 31$ On the other hand, clinical and administrative databases collect information for purposes unrelated to research, and this can create limitations related to accuracy, degree of detail, completeness of information and bias. ${ }^{29}{ }^{31}$ Regardless, linkage of these large, population-based databases offers 'an opportunity to create new data from existing sources'. ${ }^{31}$

Clinical databases with information on laboratory tests can provide insight into HIV care engagement and health outcomes among PLWH. For example, VL and CD4 laboratory tests are commonly used as proxies for HIV care visits, ${ }^{20}$ and a suppressed VL is the ultimate goal of ART and an important predictor of health and onward HIV transmission. ${ }^{12}{ }^{33}$ In Ontario, HIV diagnostic and VL testing is conducted by the Public Health Ontario Laboratory (PHOL), with laboratory test results and sociodemographic/clinical information collected on requisition/ surveillance forms inputted into centralised databases. To improve our understanding of diagnosed PLWH in Ontario, these databases were linked and used to create a population-based cohort for retrospective and prospective measurement of factors associated with health outcomes. Indeed, a paper recently published by our team presents trends in HIV cascade engagement and briefly describes the creation of this new cohort. ${ }^{34}$

In this cohort profile, we describe the integration and linkage of PHOL laboratory databases and subsequent creation of the Ontario HIV Laboratory Cohort in more detail. We also use sociodemographic data to characterise cohort participants and those lost to follow-up (LTFU), describe cohort dynamics and assess potential selection biases introduced by our exclusion criteria. Finally, we briefly summarise our findings to date on HIV cascade engagement and provide an in-depth discussion on the strengths and limitations of our cohort. In the future, our team plans to conduct further HIV cascade analyses and link to databases with information on deaths, physician billings and prescriptions in order to address cohort limitations and increase opportunities for research.

\section{COHORT DESCRIPTION}

\section{Data sources}

Public Health Ontario Laboratory information systems.

\section{HIV diagnostic testing databases}

PHOL information systems contain centralised data on HIV-positive (1985-present) and HIV-negative (1992present) diagnostic tests. All diagnostic testing conducted by healthcare providers in Ontario is done by PHOL. However, a small but unknown number of tests are conducted outside of the public health laboratory system in private laboratories for the purpose of screening organ/tissue and blood donors. These tests are not included in PHOL databases.

Sociodemographic information on individuals tested for HIV is collected on two different forms. Age, sex and HIV exposure category are documented by the ordering provider on the HIV diagnostic test requisition form. If 
an individual tests HIV-positive for the first time (ie, there is no previous HIV-positive diagnostic record in PHOL databases), the Laboratory Enhancement Program (LEP) questionnaire is sent to the provider in order to collect further information on the diagnosed individual. This includes information also collected on the diagnostic test requisition (in order to reduce missing information), as well as information not collected on the requisition (eg, race/ethnicity and country of birth). The LEP form was introduced in 1999 and race/ethnicity and country of birth were added to the questionnaire in 2009.

Diagnostic testing in the province can be conducted nominally (ie, test requisition form includes name of the person tested) or non-nominally. Non-nominal forms of testing include the use of codes or completely anonymous identifiers on the diagnostic test requisition form. Of the 485238 diagnostic HIV tests conducted in 2015, $93.3 \%$ of were nominal, $3.2 \%$ were coded and $2.9 \%$ were anonymous. ${ }^{35}$

\section{Viral load testing databases}

PHOL information systems also contain centralised data on VL tests conducted from 1996 onwards, when VL testing was first implemented in the province. In Ontario, VL testing is conducted as part of routine HIV care for diagnosed PLWH and all testing in the province is done by PHOL. Age, sex, most recent CD4 count and use of ART medications are documented by the ordering provider on VL test requisition forms. While changes to provincial public health and laboratory legislation led to anonymous VL testing being permitted from 1 July 2015 onwards, all VL tests in the database were nominal as of the end of 2015.

\section{HIV datamart}

In 2014, Public Health Ontario initiated development of an HIV datamart using PHOL laboratory information systems. This was done in order to facilitate analyses of diverse components of PHOL's HIV data for policy, planning, clinical, surveillance, public health, research and operational purposes. Development of the datamart involved the extraction of demographic, diagnostic and VL data from the specimen-based laboratory information systems and linkage of these data elements at the individual level. The datamart integrates data from both legacy and current laboratory information systems, as well as LEP data that are entered and stored in a separate relational database. The integration process ensures all fields are mapped, standardised and quality checked across the various sources and permits the creation of derived fields, such as age groups and clinically significant VL ranges. Non-clinical, quality control samples and records associated with targeted studies are excluded from the datamart.

When possible, all lab tests for an individual are linked and assigned a unique identifier in the datamart using their health insurance plan number or, if not available, custom deterministic and probabilistic matching algorithms based on other demographic information (ie, first name, last name and date of birth). Linkage of diagnostic tests to other tests (diagnostic or VL) is possible only for those tested nominally, as the requisite demographics used for linkage are not available for non-nominal diagnoses (ie, anonymous or coded).

The datamart is updated annually using incremental loads in which new individuals are added to the datamart, and new data for pre-existing individuals are added via linkage to previous results.

\section{Cohort creation}

A number of inclusion/exclusion criteria were applied to the datamart to construct a cohort of HIV-diagnosed individuals for both retrospective and prospective analyses. Individuals in the datamart are included in the cohort with the first record of a nominal HIV-positive diagnostic test or VL test. Non-nominal diagnostic tests are excluded from the cohort due to the lack identifying information necessary to permit linkage. In the absence of linkage, it is not possible to track health-related outcomes or determine whether individuals diagnosed non-nominally are duplicated within the nominal data. Of note, many individuals diagnosed non-nominally also receive a nominal diagnostic test when entering care, thus increasing the potential for duplication. Importantly, while non-nominal diagnostic tests are excluded, individuals diagnosed non-nominally are included in the cohort with a subsequent record of a nominal HIV-positive diagnostic test or VL test. Non-nominal tests are an inherent limitation of surveillance data, consistent with data sources created for similar purposes in other jurisdictions. ${ }^{22}$

In our cohort, we include individuals with a VL test only (ie, no linked nominal HIV-positive diagnostic test) in order to capture individuals who were (1) diagnosed through non-nominal testing within Ontario or (2) previously diagnosed elsewhere and then migrated to Ontario. However, individuals with no linked nominal HIV-positive diagnostic test, all undetectable VL (uVL) tests and evidence of being HIV negative (ie, record of a nominal HIV-negative diagnostic test after, on the same day as, or within 30 days before their last $\mathrm{uVL}$ test) are excluded, as they are likely HIV-negative individuals who received a VL test for diagnostic purposes. In Ontario, use of VL testing to diagnose HIV infection is not recommended/standard practice, but is possibly done by a small number of physicians because of its shorter window period.

\section{Cohort follow-up}

Individuals remain in the cohort unless administratively LTFU, defined as $>2$ consecutive years with no VL test and no VL test in later years. The LTFU rule is applied to indirectly censor for death and migration out of Ontario (out-migration), as these data are not available in PHOL databases. Other administrative studies of PLWH have also indirectly censored for death and/or out-migration, but there is no standardised approach. ${ }^{20} 36$ All individuals in our cohort not assessed as LTFU are defined 
as diagnosed PLWH in Ontario. Later in this paper, we provide a summary of indirect censorship approaches used elsewhere and also describe planned linkage to death/migration data.

Importantly, individuals assessed as LTFU are not permanently censored but can re-enter the cohort with a subsequent record of a nominal HIV-positive diagnostic or VL test. Returning individuals are retrospectively included in the cohort as a diagnosed PLWH for the years in which they had no record of a VL test. We selected the 2-year LTFU criteria given that this would capture most individuals in our cohort experiencing a known gap in care (ie, no VL test in $\geq 1$ year but record of a VL in a later year). Between 2000 and 2014, the median (IQR) duration of known gaps in care was $1.4(1.1,2.0)$ years.

\section{Newly diagnosed sample}

We also used the datamart to create a sample of individuals who were newly diagnosed with HIV in Ontario and not outside of the province (ie, were not previously diagnosed elsewhere and later migrated to Ontario). This sample was created for two reasons. First, newly diagnosed individuals can differ from prevalent cases in several ways that may affect HIV care engagement (eg, prevalent cases have been diagnosed for a longer period of time and include those diagnosed outside of Ontario) and, consequently, others ${ }^{38}$ have recommended the construction of separate HIV cascades for each population. Second, such samples are necessary for measurement of longitudinal HIV care indicators, including time from diagnosis to linkage to care and viral suppression. ${ }^{20} 3940$

For the newly diagnosed sample, we include individuals with a nominal HIV-positive diagnostic test and exclude non-nominal tests, as done for the cohort. Unlike the cohort, individuals with a VL test only (ie, no linked nominal HIV-positive diagnostic test) are excluded, as the lack of a diagnostic test record precludes measurement of 'time-from-diagnosis' indicators. Individuals with laboratory evidence of a previous diagnosis prior to their first PHOL nominal HIV-positive diagnostic test record are also excluded. This evidence includes having had a (1) detectable VL test or documented CD4 result prior to their first HIV-positive diagnostic test or (2) a first VL test after HIV diagnosis that was virally suppressed $(<200$ copies $/ \mathrm{mL})$.

\section{Patient and public involvement in research}

This research was done without patient involvement due to the retrospective, administrative nature of the cohort. However, cohort analyses and dissemination of findings are guided by ongoing advice from a community advisory committee: people working in the community-based HIV service sector and HIV clinics whose input helps ensure that reports and other products support collective efforts and impact on neighbourhoods, communities and organisations across the province.

\section{FINDINGS TO DATE}

\section{HIV datamart}

To the end of 2015, a total of 8532559 diagnostic test records (includes nominal, non-nominal, HIV-negative and HIV-positive tests) and $595111 \mathrm{VL}$ test records were extracted from the PHOL information systems. After excluding non-clinical tests (2575 diagnostic and 40946 $\mathrm{VL}$ ) and linking diagnostic/VL tests at the individual level, the datamart contained information on 4603801 unique individuals and 985152 non-nominal diagnostic tests.

\section{Cohort creation}

There were a total of $40372 \mathrm{HIV}$-positive diagnostic tests and 23851 individuals with $\geq 1 \mathrm{VL}$ test in the datamart as of the end of 2015 and therefore potentially eligible to enter the cohort (figure 1). Overall, 18683 (46.3\%) HIV-positive diagnostic tests were non-nominal and excluded. The proportion of HIV-positive diagnostic tests that were non-nominal decreased from $48.9 \%$ in 2000 to $15.0 \%$ in 2015 (figure 2), due to a decline in coded HIV-positive tests. Linkage of nominal HIV-positive diagnostic and VL tests resulted in 30534 unique individuals. Of these, a total of 2300 had no linked diagnostic test and all uVL tests, of whom 947 had a record of an HIV-negative test after their last uVL test and were excluded.

Overall, the cumulative laboratory cohort by end of 2015 included 29587 unique individuals with diagnosed HIV. Of individuals who entered the cohort following the introduction of VL testing in 1996, the majority (61.9\%) had an HIV-positive diagnostic test linked to $\geq 1$ VL test, $32.6 \%$ had a VL test only (ie, no linked nominal HIV-positive diagnostic test) and $5.5 \%$ had an HIV-positive diagnostic test only (ie, no linked VL test).

\section{Cohort follow-up}

Between 1996 and 2015, the 29587 cohort participants were followed for a total of 229302 person-years and received $552855 \mathrm{VL}$ tests. The number of diagnosed PLWH (ie, cohort individuals not assessed as LTFU) almost doubled between 2000 and 2015, increasing from 8859 to 16110 (figure 3A). This increase was likely due to a combination of ongoing new HIV diagnoses and improved life expectancy of diagnosed PLWH.

Cohort dynamics are shown in figure 3 . The median number of people entering and exiting the cohort each year was 975 (range: 855-1116) and 482 (range: 444-604), respectively (figure 3B). Over the past decade, the percent of cohort participants who were LTFU each year was relatively stable and ranged from $3.4 \%$ to $4.0 \%$ (figure 3C).

\section{Assessment of bias}

To explore potential selection biases introduced by the exclusion of non-nominal tests and the application of our LTFU criteria, we compared participant characteristics by (1) type of diagnostic test (nominal vs non-nominal) and (2) VL linkage status (nominal diagnostic test 


\section{Confirmed HIV-positive diagnostic test (1985-2015)}

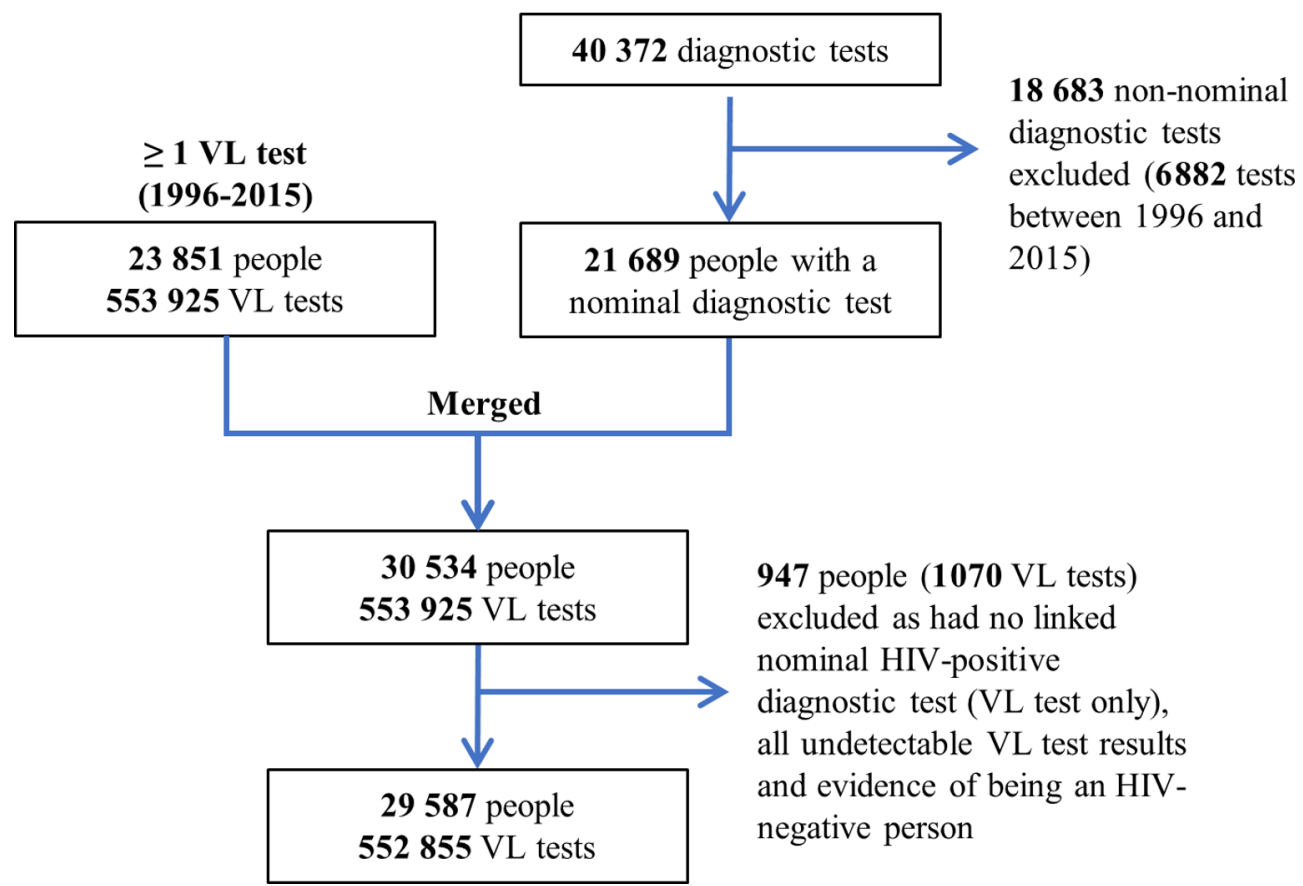

Figure 1 Flow diagram for the creation of the Ontario HIV Laboratory Cohort from the Public Health Ontario Laboratory HIV datamart. Non-nominal forms of testing include the use of coded or completely anonymous identifiers. Evidence of being an HIV-negative person is defined as record of a nominal HIV-negative diagnostic test after, on the same day as, or within 30 days before last undetectable viral load test. VL, viral load (from Wilton et $a^{3{ }^{34}}$ ).

linked vs not linked to a VL test). We explored the latter as all individuals with no linked VL test were judged as LTFU and removed from the cohort after 2 years, which could bias the cohort by excluding individuals who never link to care after diagnosis but continue residing in the province.

Differences in characteristics of HIV-positive diagnostic tests by type of identifier (nominal vs non-nominal) are shown in table 1 . Of note, non-nominal diagnoses were more likely to be men who have sex with men (MSM; $70.9 \%$ vs $40.8 \%, \mathrm{p}<0.0001)$ and diagnosed between 1996 and 2000 (40.5\% vs 18.6\%, p<0.0001). Non-nominal diagnoses were less likely to be from an HIV-endemic country ( $7.0 \%$ vs $19.3 \%, \mathrm{p}<0.0001)$, Black $(14.0 \%$ vs. $27.6 \%, \mathrm{p}<0.0001)$ and heterosexual $(11.8 \%$ vs $19.4 \%, \mathrm{p}<0.0001)$.

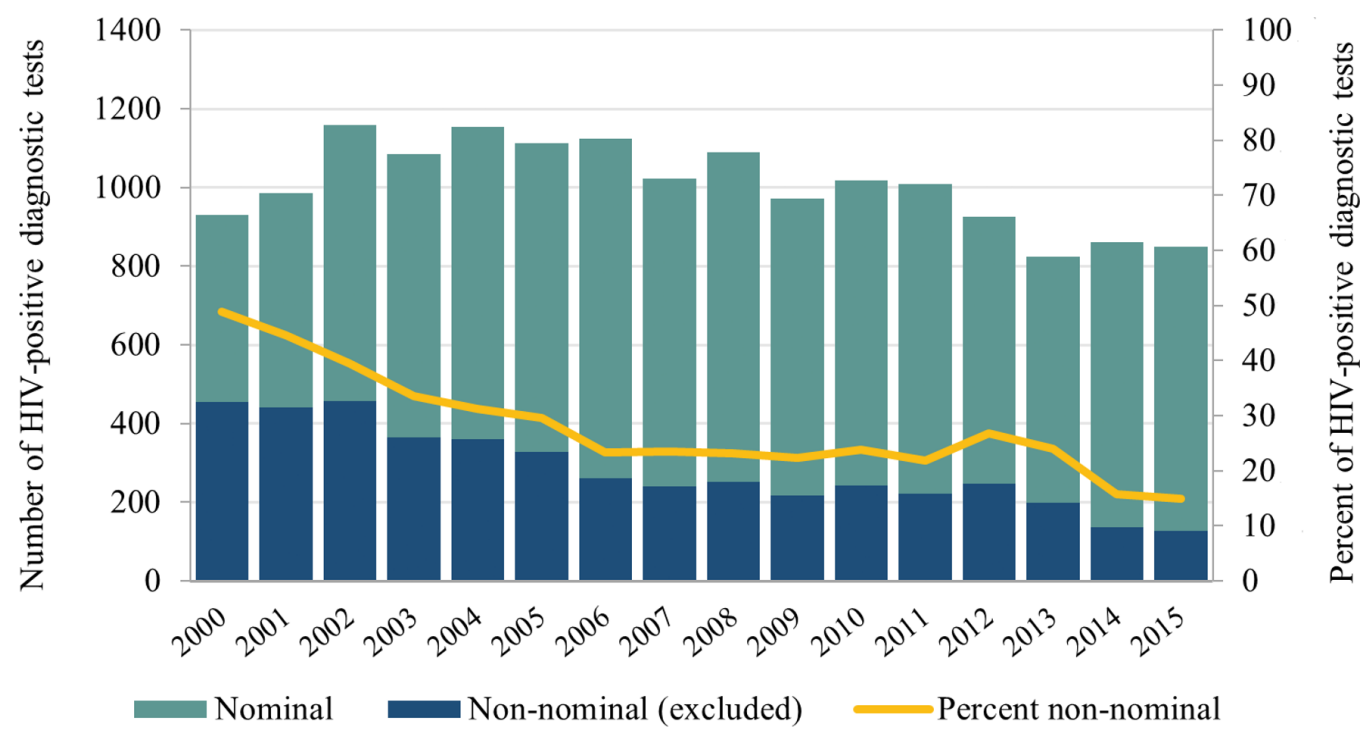

Figure 2 Number and percent of HIV-positive diagnostic tests by type of identifier (nominal vs non-nominal), Public Health Ontario Laboratory HIV datamart, 2000-2015. Non-nominal forms of testing include the use of coded or completely anonymous identifiers. See online supplementary file 3 for underlying data. 

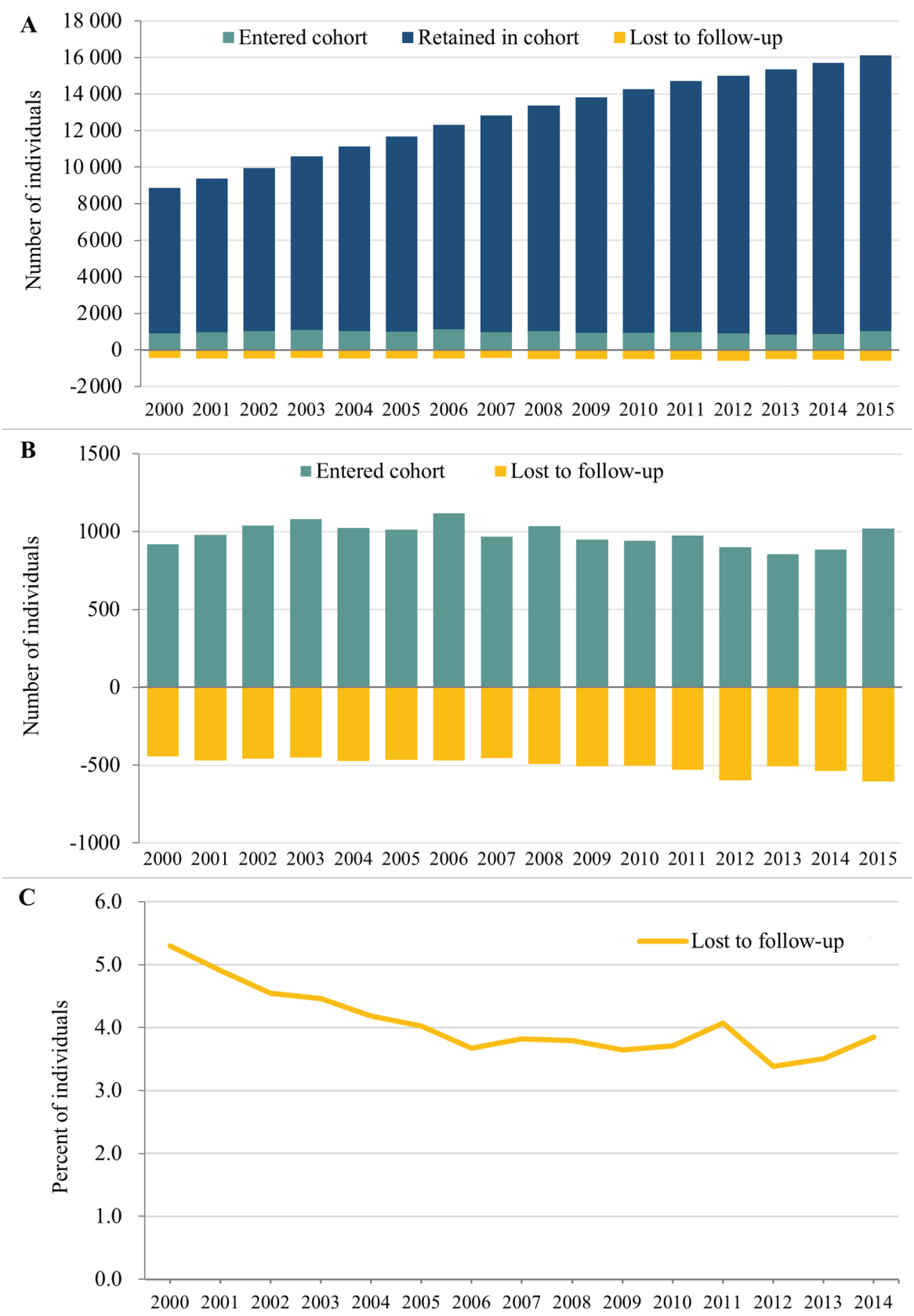

Figure 3 Ontario HIV Laboratory Cohort dynamics, 2000-2015. (A) Annual number of individuals who entered cohort, were retained in cohort or were LTFU. (B) Annual number of individuals who entered cohort or were LTFU. (C) Annual percent of participants who were LTFU. (B) is the same as (A) except 'Retained in cohort' was removed. See online supplementary file 3 for underlying data. LTFU, lost to follow-up (no VL test for $>2$ years and no VL test in later years).

The proportion of nominally diagnosed individuals who did not have a linked VL test was relatively stable over the past decade and ranged from $5.0 \%$ to $8.2 \%$ (figure 4 ). Differences in nominally diagnosed individuals by VL linkage status (linked vs not linked to a VL test) are shown in table 1. Of note, individuals not linked to a VL test were more likely to be diagnosed between 1996 and 2000 (34.0\% vs $16.9 \%, \mathrm{p}<0.0001)$ and missing information on sex, age and health insurance plan number (identifiers used to link records within the datamart, all $\mathrm{p}<0.0001$ ). Those without a linked VL test were less likely to be MSM ( $30.0 \%$ vs $41.7 \%, \mathrm{p}<0.0001)$.

\section{Cohort sociodemographic profile}

In 2015, the majority of the 16110 diagnosed PLWH in the cohort were male $(79.6 \%)$ or aged $\geq 45$ years $(62.6 \%)$ (table 2). Between 2000 and 2015, the number of males and females increased by 1.7-fold and 2.5-fold, respectively (figure $5 \mathrm{~A}$ ). The percent of diagnosed PLWH who were female increased from $15.0 \%$ in 2000 to $20.0 \%$ in 2008, remaining relatively stable thereafter (figure 5B).

Trends varied by age category (figure 5C,D). Between 2000 and 2015, there was an overall ageing of the cohort population: the most common age category shifted from 35-44 to 45-54, the number of PLWH who were 
Table 1 Comparison of (1) HIV-positive diagnostic tests by type of identifier (nominal vs non-nominal) and (2) nominally diagnosed individuals by VL linkage status (linked vs not linked to a VL test)

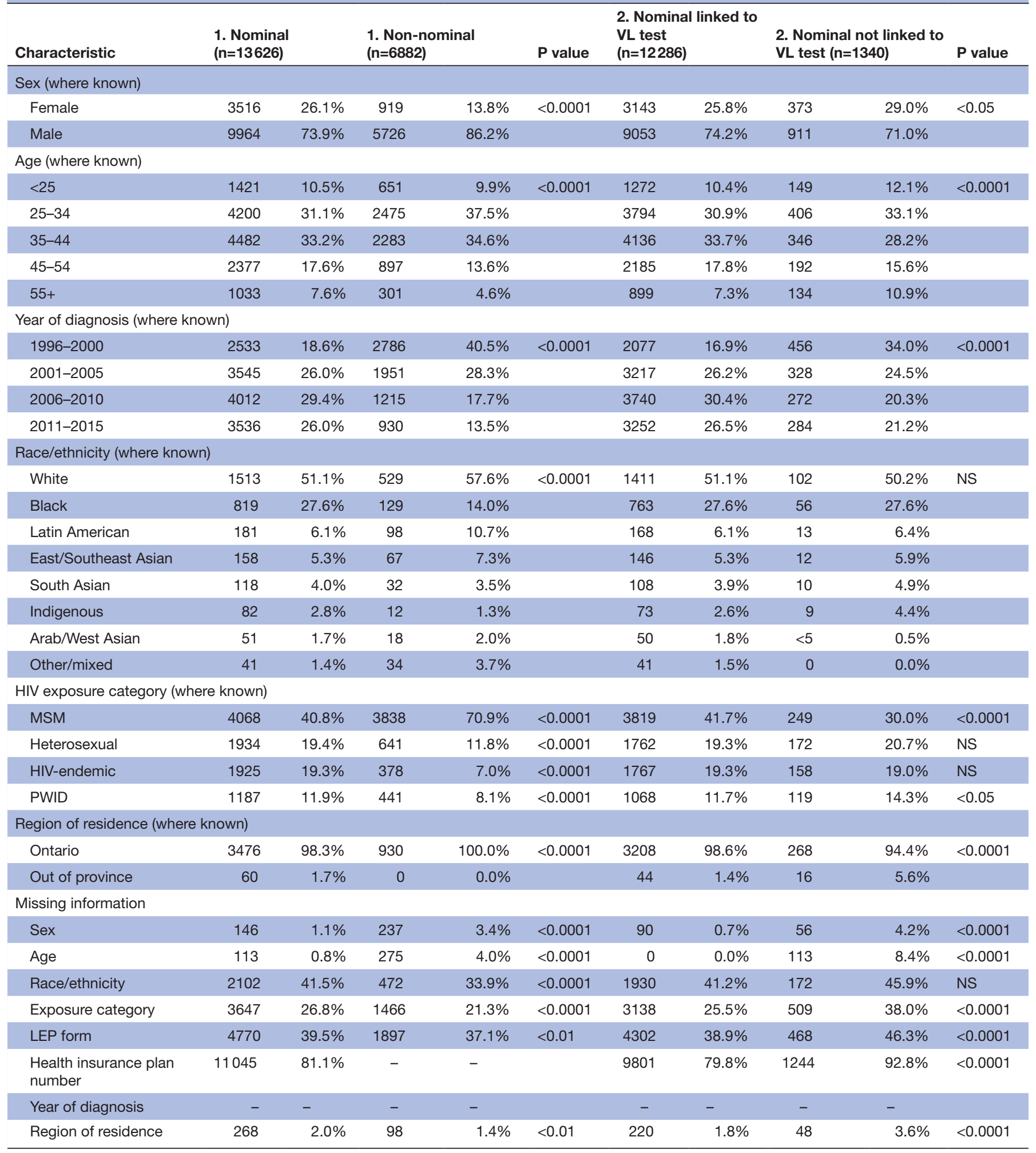

Comparisons limited to the years 1996-2015. All characteristics mutually exclusive, except for MSM and PWID exposure categories. Nominal and nonnominal columns may include some of the same individuals, as some people diagnosed non-nominally received a nominal diagnosis when entering care. Health insurance number was collected on diagnostic tests from 2010 onwards (missing 50\% of the time) and VL tests from 1996 onwards (missing 3.5\% of the time). Missing information was greater for 'Diagnosed PLWH in cohort, 2015' as this column includes participants with a VL test only (no linked nominal HIV-positive test) and the VL test requisition does not collect information on exposure category, race/ethnicity or period of diagnosis. $\chi^{2}$ tests were used to compare characteristics (continuity-adjusted tests used for $2 \times 2$ comparisons). NS, not significant at $p<0.05$. LEP, Laboratory Enhancement Program; LTFU, lost to follow-up; MSM, men who have sex with men; PLWH, people living with HIV; PWID, people who use injection drugs; VL, viral load. 


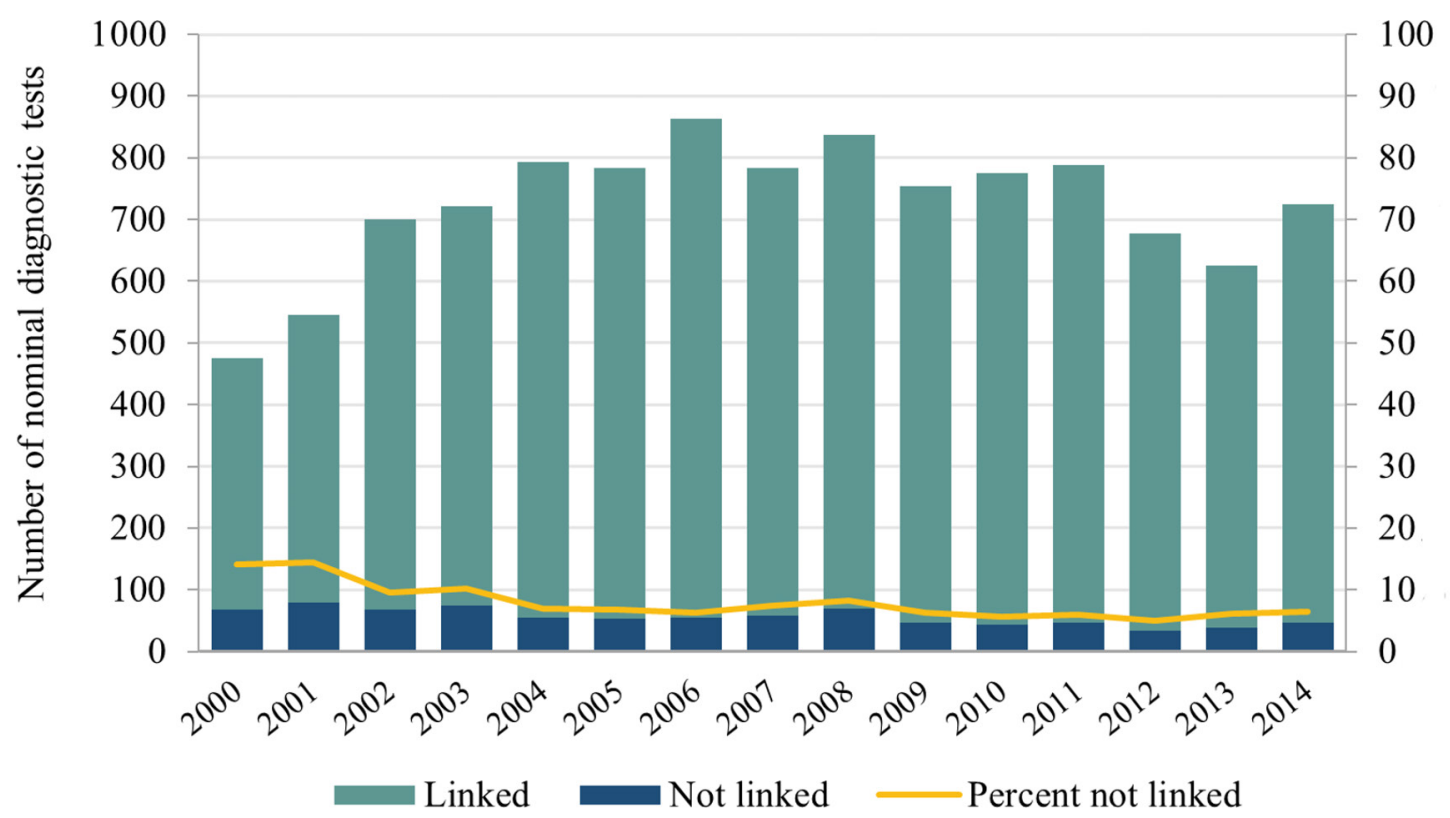

100

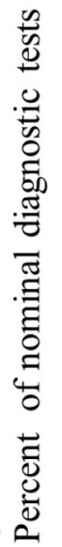

Figure 4 Number and percent of nominal HIV-positive diagnostic tests by VL linkage status (linked vs not linked to a VL test), Public Health Ontario Laboratory HIV datamart, 2000-2014. The year 2015 was removed as many individuals diagnosed in this year may not have had time to link to care and receive a VL test. See online supplementary file 3 for underlying data. VL, viral load.

$45+$ increasedalmost four-fold (from 2551 to 10063 ) and the percent $45+$ doubled from $29.1 \%$ to $62.6 \%$. This ageing is consistent with observations elsewhere and has implications for the provision of appropriate HIV care to ensure healthy ageing. ${ }^{41}$

Information on race/ethnicity and exposure category was missing for $83.5 \%$ and $49.3 \%$ of cohort participants, respectively (table 2 ). Where this information was known, the most common race/ethnicity in 2015 was White $(51.4 \%)$, followed by Black (26.9\%), and the most common exposure category was MSM $(46.4 \%)$ followed by heterosexual $(18.5 \%)$. Due to the extent of missing information, trends over time are not presented for these characteristics and the comparisons in tables 1 and 2 should be interpreted with caution.

Characteristics of individuals recently LTFU (ie, individuals who were in the cohort in 2014 but not in 2015) are shown in table 2. Compared with individuals who were still in the cohort in 2015 , those recently LTFU were less likely to be MSM $(34.2 \%$ vs $46.4 \%, \mathrm{p}<0.0001)$ and more likely to be Black ( $40.6 \%$ vs $26.9 \%, \mathrm{p}=0.01)$, from an HIV-endemic country ( $25.6 \%$ vs $16.3 \%, \mathrm{p}<0.0001)$, a person who injects drugs $(16.3 \%$ vs $10.8 \%, \mathrm{p}=0.004)$ and missing identifying information used to link tests. The latter finding suggests that inability to link tests-rather than death or out-migration-may be responsible for some LTFU.

\section{Newly diagnosed sample creation and follow-up}

There were a total of 19386 HIV-positive diagnostic tests between 1996 and 2015, of which 5976 (30.8\%) were non-nominal and excluded from the newly diagnosed sample (online supplementary file 1 shows flow diagram). Of the remaining 13410 nominal diagnoses, we excluded
3797 (28.3\%) who had evidence of being previously diagnosed. Overall, 9613 individuals were included in our newly diagnosed sample (8173 individuals from 2000 onwards). Between 2000 and 2015, the median annual number of newly diagnosed individuals entering the sample was 529 (range: 368-599) (online supplementary file 2).

\section{HIV care cascade engagement}

Our study team has published two technical reports and a peer-reviewed manuscript presenting HIV cascade indicators overall and by sex, age and geography. ${ }^{34} 4344$ Between 2000 and 2015, the percent of diagnosed PLWH who were in care ( $\geq 1 \mathrm{VL}$ test in a given year) increased from $81.3 \%$ to $87.3 \%$, on ART (as documented on last VL test requisition in a given year-individuals with missing data assumed to be on treatment if virally suppressed) increased from $55.1 \%$ to $81.1 \%$ and virally suppressed $(<200$ copies $/ \mathrm{mL}$ on last VL test in a given year) increased from $40.7 \%$ to $79.5 \%$. Between 2000 and 2014, the percent of newly diagnosed individuals who linked to care within 3 months of diagnosis and achieved viral suppression within 6 months increased from $67.4 \%$ to $81.8 \%$ and from $22.0 \%$ to $41.6 \%$, respectively. In general, cascade indicators were lower for females and people of younger ages.

The traditional cascade diagram for the year 2015 is shown in figure 6. Of note, our technical reports and manuscript present upper and/or lower bounds for each indicator using alternative definitions/assumptions. ${ }^{344344}$ These alternatives were based on aspects unique to our data source and the range of definitions adopted by other researchers. While several publications have reviewed and summarised HIV care cascade estimates from other jurisdictions, ${ }^{204546}$ comparisons should be made with caution 
Table 2 Comparison of diagnosed PLWH in cohort to individuals recently lost to follow-up, Ontario HIV Laboratory Cohort, 2015

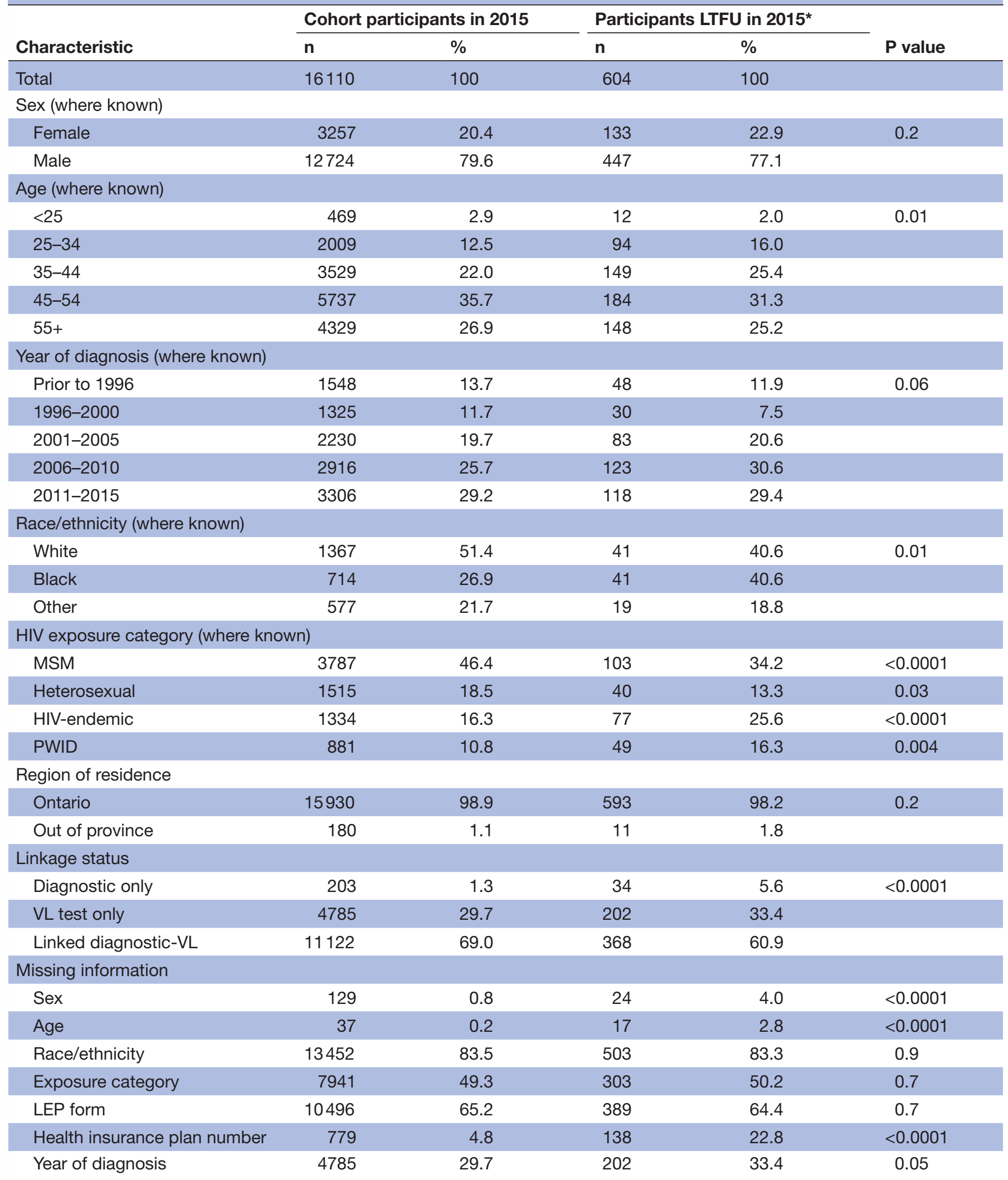

$\chi^{2}$ tests were used to compare characteristics (continuity-adjusted tests used for $2 \times 2$ comparisons). See table 1 notes for additional details. *Individuals who were in the cohort in 2014 but not in 2015.

LEP, Laboratory Enhancement Program; LTFU, lost to follow-up; MSM, men who have sex with men; PLWH, people living with HIV; PWID, people who use injection drugs; VL, viral load. 

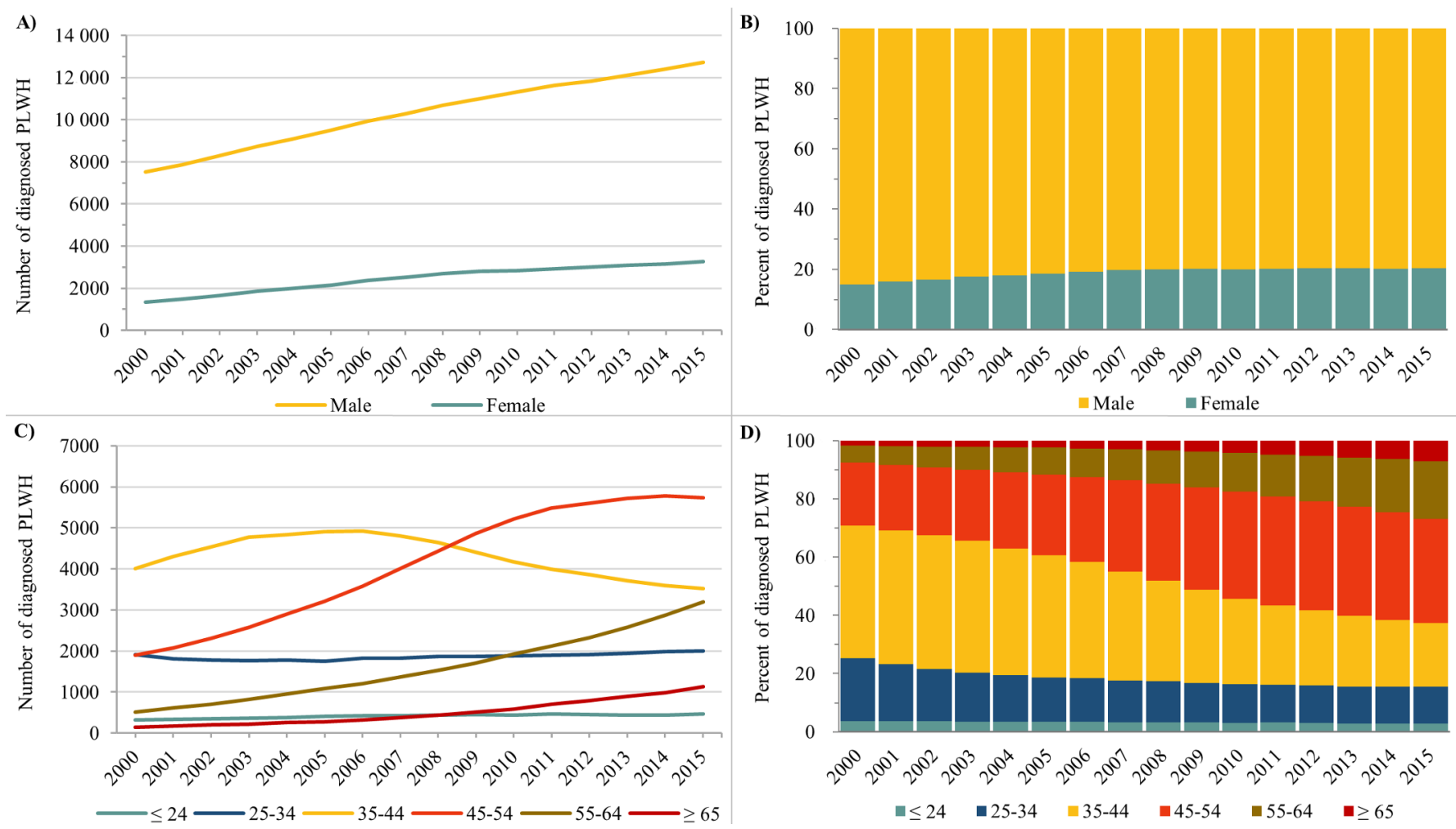

Figure 5 Trends in sex and age of diagnosed PLWH in the Ontario HIV Laboratory Cohort, 2000-2015. (A) Number of diagnosed PLWH by sex. (B) Percent of diagnosed PLWH by sex. (C) Number of diagnosed PLWH by age category. (D) Percent of diagnosed PLWH by age category. Diagnosed PLWH=cohort participants not lost to follow-up. See online supplementary file 3 for underlying data. PLWH, people living with HIV.

due to the varied indicator definitions, study methodologies and data sources used across studies.

While Ontario HIV Laboratory Cohort data is longitudinal, our cascade analyses conducted to date have been mostly cross-sectional in nature. ${ }^{34}$ These analyses do not consider the dynamic nature of HIV care or population changes over time. ${ }^{21} 36$ Longitudinal approaches and non-linear cascade frameworks will inform future analyses.

\section{Strengths and limitations}

We created a cohort of people living with diagnosed HIV in the Canadian province of Ontario for both retrospective and prospective measurement of factors associated with health outcomes (eg, HIV cascade engagement), as well as linkage to additional databases. Our cohort is population based, was created using centralised diagnostic and VL laboratory databases linked at the individual level, and contained information on approximately 30000 people with diagnosed HIV as of the end of 2015.

The Ontario HIV Laboratory Cohort fills an important gap in available data sources in Ontario, as other existing HIV-related sources are either not population based, limited to PLWH already in care and/or do not contain information on laboratory test results (eg, viral suppression). ${ }^{27} 47$ To the best of our knowledge, the Ontario HIV Laboratory Cohort is one of only a few published population-based cohorts of diagnosed PLWH with individual-level linkage across the HIV care cascade (from diagnosis to viral suppression). ${ }^{20} 2132$ In addition to the cohort, we created a sample of individuals who were newly diagnosed with HIV in Ontario in order to measure longitudinal cascade indicators (eg, time from diagnosis to linkage to care).

While our cohort includes the vast majority of diagnosed PLWH in Ontario (due to the centralised nature of our data sources), our indirect approach to censoring for death and out-migration ( $>2$ years with no VL test) has likely led to the inappropriate removal of some individuals. Other population-based studies of diagnosed PLWH have also censored for death and/or out-migration indirectly, but there is no standardised approach. ${ }^{20}$ For example, a British Columbia (BC) cohort removes individuals with no healthcare use for $\geq 1.5$ years, ${ }^{22}$ a New York City study removed individuals with no VL test for $\geq 5$ years $^{48}$ and a study ${ }^{1}$ of an Ontario-based administrative cohort ${ }^{47}$ removed individuals with no healthcare use for $\geq 7$ years ( $T$ Antoniou, personal communication). Importantly, individuals who return to care after being LTFU are re-entered into our cohort and retrospectively included as diagnosed PLWH for the intervening years.

Our indirect approach to censoring for death and out-migration may require further validation and refinement. However, it is reassuring that the annual percent of individuals who were LTFU in our study was very similar to the $\mathrm{BC}$ cohort (as censored via linkage to vital statistics and removal of individuals with no healthcare use for $\geq 1.5$ 


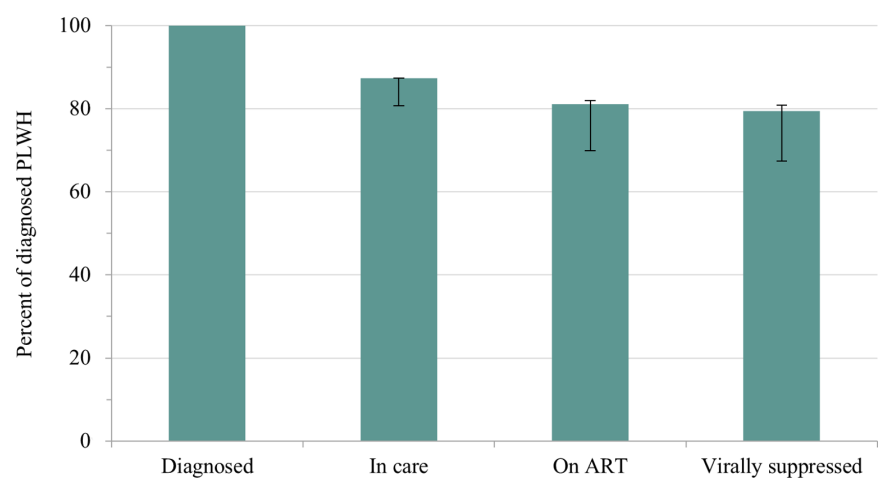

Figure 6 Percent of diagnosed PLWH engaged in the HIV care cascade, Ontario HIV Laboratory Cohort, 2015. Solid column bar represents main estimate. Black brackets represent upper and lower bounds. Diagnosed PLWH refers to cohort participants not lost to followup. See references ${ }^{34} 434$ for indicator definitions. ART, antiretroviral treatment; PLWH, people living with HIV.

years).$^{22}$ On the other hand, the number of individuals in our cohort was lower compared to an analysis of a population-based Ontario cohort created using physician billing claims. ${ }^{47}$ In this analysis, deaths and out-migration were censored via linkage to vital statistics and removal of individuals with no healthcare use for $\geq 7$ years, respectively, and $15107 \mathrm{HIV}$-diagnosed individuals were estimated to be living in Ontario in 2009/2010. ${ }^{1}$ This estimate was higher than the 14255 in our cohort in 2010 . The varied LTFU criteria (2 vs 7 years) may explain the difference between the two cohorts.

We plan to link our cohort to other administrative data in order to address censorship limitations and validate/ enhance our LTFU rule. Vital statistics will allow for direct death censorship, while non-HIV physician billing data could be used to enhance our LTFU rule and better determine whether an individual is still living in the province. Information on registration status for Ontario's healthcare plan could improve detection of out-migration (ie, cancellation likely indicates an individual has left the province).

A small number of people who reside outside of Ontario were included in our cohort. In 2015, $180(1.1 \%)$ of the 16110 diagnosed PLWH were living outside of Ontariothe majority $(\sim 85 \%)$ of whom were residents of Quebec (a neighbouring province). Out-of-province individuals were included in our HIV cascade analyses as their data are reflective of the quality of HIV care provided in Ontario. However, these individuals may be removed from future analyses depending on the research question. Of note, data on individuals living in Ontario but receiving HIV care elsewhere (eg, Quebec) are not adequately captured in the cohort.

Our cohort may be biased towards not including individuals who never connect to care after diagnosis, as these individuals are either not adequately retained (ie, those diagnosed nominally are removed from our cohort after 2 years) or not included at all (ie, those diagnosed non-nominally never enter our cohort). Importantly, the number of individuals who never connect to care after diagnosis is likely small. While about $6 \%$ of nominally diagnosed participants in our cohort did not have a linked VL test, these individuals were more likely to be missing information vital to data linkage processes. This suggests lack of a linked VL test was, in some cases, due to insufficient identifying information rather than lack of linkage to care. As such, many of these individuals may actually be in the cohort as participants with a VL test but no linked diagnostic test.

There were a large number of non-nominal HIV-positive diagnostic test records and their exclusion may have introduced selection bias into our cohort. However, while non-nominal tests are excluded from our cohort, our assumption is that the majority of non-nominally diagnosed individuals will present for HIV care and be included in the cohort once they receive a nominal VL test. Nevertheless, non-nominally diagnosed individuals who never connect to care are not included in our cohort (as discussed above) and our newly diagnosed sample excludes individuals with a VL test only (and is therefore less likely to capture non-nominally diagnosed individuals). This likely introduces bias into the newly diagnosed sample, as we identified several sociodemographic differences between nominal and non-nominal diagnoses. In particular, non-nominally diagnosed individuals in our databases were much more likely to be men who have sex with men, and studies have shown that this population experience better engagement in HIV care compared with heterosexual men and women. ${ }^{28}$ Of note, the percent of HIV-positive diagnostic tests that were non-nominal has decreased markedly over time, reducing the potential for bias in more recent years.

The laboratory databases used to create our cohort include most, but not all, of the information commonly used to measure engagement in HIV care. CD4 tests are a commonly used proxy for HIV care visits, ${ }^{20}$ but CD4 testing is not centralised in Ontario, and a single population-based data source does not exist. ART use and adherence are also critical components of HIV cascade measurement, but our databases lack prescription/pharmacy records. While use of ART medications and most recent $\mathrm{CD} 4$ count are recorded on VL test requisitions forms by the ordering provider, they are missing for $20 \%$ and $43 \%$ of VL test requisitions, respectively. Planned linkage to prescription data may improve measurement of ART-related indicators.

Completeness of participant sociodemographic information varied widely. While age, sex and geography were very complete, the extent of missing information was much higher for race/ethnicity and HIV exposure category. Missing information was due to a combination of factors, including (1) providers not filling out diagnostic requisition forms, (2) race/ethnicity not being collected prior to 2009 and (3) race/ethnicity and HIV exposure category only being collected on diagnostic requisition forms and thus missing for participants with no linked diagnostic test (approximately 25\% of participants). 
This missing information limits some of the comparisons made in this paper and will also limit our ability to fully assess care and health indicators by sociodemographic characteristics.

In conclusion, our population-based cohort of diagnosed PLWH in Ontario — created using centralised laboratory databases-is, in many ways, an optimal data source for measuring engagement in HIV care. Planned linkage to administrative databases with information on deaths, migration, physician billing claims and prescriptions will help address cohort limitations and create new opportunities for research.

\section{COLLABORATION}

If interested in collaborating on cohort analyses, please contact lab.data@oahpp.ca. The Ontario Agency for Health Protection and Promotion (operating as Public Health Ontario) is a government agency in Ontario, Canada, and is authorised to collect, use and disclose personal health information, including the data used in this study, under the Personal Health Information and Protection Act, 2004, Health Protection and Promotion Act and the Laboratory and Specimen Collection Centre Licensing Act. Public Health Ontario is permitted to disclose the data used in this study with other researchers, provided that the researcher and the proposed cohort research project meet specific requirements of Ontario privacy laws, Public Health Ontario's privacy and ethics policies, and the most current version of the Tri-Council Policy Statement: Ethical Conduct for Research Involving Humans, as applicable. While deidentified or aggregate-level data may be shared with fewer restrictions, all proposals require a privacy review, an assessment of available resources at Public Health Ontario to conduct the analyses and may also require a data sharing agreement. Information about PHO's data access request process is available online (https://www.publichealthontario.ca/ en/About/Pages/Data.aspx).

\section{Author affiliations}

${ }^{1}$ Public Health Ontario, Toronto, Ontario, Canada

${ }^{2}$ Ontario HIV Treatment Network, Toronto, Ontario, Canada

${ }^{3}$ Public Health Agency of Canada, Ottawa, Ontario, Canada

${ }^{4}$ Division of Clinical Public Health, Dalla Lana School of Public Health, University of Toronto, Toronto, Ontario, Canada

${ }^{5}$ Division of Social and Behavioural Health Sciences, Dalla Lana School of Public Health, University of Toronto, Toronto, Ontario, Canada

${ }^{6}$ Clinical Prevention Services, British Columbia Centre for Disease Control,

Vancouver, British Columbia, Canada

${ }^{7}$ School of Population and Public Health, University of British Columbia, Vancouver, British Columbia, Canada

Acknowledgements Some of the data presented in the tables and figures in this paper were included as supplementary information in a previous manuscript published by our research team (Wilton J et al. PLoS ONE 2019;14;e0210096). The creation of the Ontario HIV Laboratory Cohort is also partially described in this other manuscript (Wilton J et al. PLOS ONE 2019;14;e0210096). We thank the individuals who make up the Ontario HIV Laboratory Cohort and the healthcare providers for completing the test requisitions and other surveillance forms. All data were provided to the Ontario HIV Epidemiology and Surveillance Initiative (OHESI) in deidentified aggregate format by the Public Health Ontario Laboratory with significant support from Public Health Ontario's database team, in particular Jane Xiong and loana Belchita. OHESI is a collaboration involving the AIDS Bureau of the Ontario Ministry of Health and Long-Term Care (MOHLTC), Public Health Ontario (PHO), the Public Health Agency of Canada (PHAC) and the Ontario HIV Treatment Network (OHTN).

Collaborators OHESI members include Jean Bacon, Beth Rachlis, James Wilton, Lucia Light, Abigail Kroch, Madison Giles (OHTN); Joanne Lush, Ken English (MOHLTC); Doug Sider, Michelle Murti, Vanessa Allen, Tony Mazzulli, Mike Mendaglio, Alex Marchand-Austin, Juan Liu (PHO); Ashleigh Sullivan and Chris Archibald (PHAC).

Contributors MGilbert, DS and AS conceived the study. JL, AS, AM-A, MGilbert, $\mathrm{JW}, \mathrm{BR}$ and $\mathrm{LL}$ planned the analyses and JL conducted the analysis. Project administration/supervision was provided by MGiles, MGilbert, AEK, DS and AM-A. JW and MGilbert wrote the manuscript. All authors critically revised the manuscript and contributed to interpretation of the data. All authors read and approved the final version of the manuscript.

Funding The authors have not declared a specific grant for this research from any funding agency in the public, commercial or not-for-profit sectors.

Disclaimer The opinions, results and conclusions are those of the authors only. No endorsement by the Ontario HIV Treatment Network, Public Health Ontario, Public Health Agency of Canada or Ontario Ministry of Health and Long-Term Care is intended or should be inferred.

Competing interests None declared.

Patient consent for publication Not required.

Ethics approval This applied research study was approved by the Ethics Review Board at Public Health Ontario (\#2016-002.01).

Provenance and peer review Not commissioned; externally peer reviewed.

Data sharing statement All data described in this manuscript are available at the aggregate level within the manuscript tables and Supplementary files. Please see the 'Collaboration' section of the manuscript for more information on data sharing.

Open access This is an open access article distributed in accordance with the Creative Commons Attribution Non Commercial (CC BY-NC 4.0) license, which permits others to distribute, remix, adapt, build upon this work non-commercially, and license their derivative works on different terms, provided the original work is properly cited, appropriate credit is given, any changes made indicated, and the use is non-commercial. See: http://creativecommons.org/licenses/by-nc/4.0/.

\section{REFERENCES}

1. Antoniou T, Zagorski B, Bayoumi AM, et al. Trends in HIV prevalence, new HIV diagnoses, and mortality among adults with HIV who entered care in Ontario, 1996/1997 to 2009/2010: a populationbased study. Open Med 2013;7:e98-106.

2. Croxford S, Kitching A, Desai S, et al. Mortality and causes of death in people diagnosed with HIV in the era of highly active antiretroviral therapy compared with the general population: an analysis of a national observational cohort. Lancet Public Health 2017;2:e35-46.

3. Poorolajal J, Hooshmand E, Mahjub H, et al. Survival rate of AIDS disease and mortality in HIV-infected patients: a meta-analysis. Public Health 2016;139:3-12.

4. Patterson S, Cescon A, Samji H, et al. Life expectancy of HIVpositive individuals on combination antiretroviral therapy in Canada. BMC Infect Dis 2015;15:274.

5. Pharris A, Quinten C, Tavoschi L, et al. Trends in HIV surveillance data in the EU/EEA, 2005 to 2014: new HIV diagnoses still increasing in men who have sex with men. Euro Surveill 2015;20:30071.

6. Centre for Disease Control. HIV surveillance report. 2016 https:// www.cdc.gov/hiv/pdf/library/reports/surveillance/cdc-hivsurveillance-report-2016-vol-28.pdf (accessed 6 Dec 2017).

7. Bourgeois AC, Edmunds M, Awan A, et al. HIV in CanadaSurveillance Report, 2016. Can Commun Dis Rep 2017;43:248-56.

8. Samji H, Cescon A, Hogg RS, et al. Closing the gap: increases in life expectancy among treated HIV-positive individuals in the United States and Canada. PLoS One 2013;8:e81355.

9. May M, Gompels M, Delpech V, et al. Impact of late diagnosis and treatment on life expectancy in people with HIV-1: UK Collaborative HIV Cohort (UK CHIC) Study. BMJ 2011;343:d6016.

10. Antiretroviral Therapy Cohort Collaboration. Survival of HIV-positive patients starting antiretroviral therapy between 1996 and 2013: a collaborative analysis of cohort studies. Lancet HIV 2017;4:e349-56. 
11. Cohen MS, Chen YQ, McCauley M, et al. Prevention of HIV1 infection with early antiretroviral therapy. $N$ Engl J Med 2011;365:493-505.

12. Rodger AJ, Cambiano V, Bruun T, et al. Sexual Activity Without Condoms and Risk of HIV Transmission in Serodifferent Couples When the HIV-Positive Partner Is Using Suppressive Antiretrovira Therapy. JAMA 2016;316:171-81.

13. Gardner EM, McLees MP, Steiner JF, et al. The spectrum of engagement in HIV care and its relevance to test-and-treat strategies for prevention of HIV infection. Clin Infect Dis 2011;52:793-800.

14. Bing EG, Burnam MA, Longshore $D$, et al. Psychiatric disorders and drug use among human immunodeficiency virus-infected adults in the United States. Arch Gen Psychiatry 2001;58:721-8.

15. Sherbourne CD, Hays RD, Fleishman JA, et al. Impact of psychiatric conditions on health-related quality of life in persons with HIV infection. Am J Psychiatry 2000;157:248-54.

16. Ferlatte $\mathrm{O}$, Hottes TS, Trussler T, et al. Evidence of a syndemic among young Canadian gay and bisexual men: uncovering the associations between anti-gay experiences, psychosocial issues, and HIV risk. AIDS Behav 2014;18:1256-63.

17. Skarbinski J, Rosenberg E, Paz-Bailey G, et al. Human immunodeficiency virus transmission at each step of the care continuum in the United States. JAMA Intern Med 2015;175:588-96.

18. UNAIDS. 90-90-90 An ambitious treatment target to help end the AIDS epidemic. http://www.unaids.org/sites/default/files/media asset/90-90-90 en 0.pdf (accessed 6 Dec 2017).

19. Ontario Advisory Committee on HIV/AIDS. HIV/AIDS Strategy to 2026: Focusing Our Efforts - Changing the Course of the Prevention, Engagement and Care Cascade in Ontario. http://www.health.gov.on ca/en/pro/programs/hivaids/docs/oach_strategy_2026.pdf (accessed 6 Dec 2017).

20. Medland NA, McMahon JH, Chow EP, et al. The HIV care cascade: a systematic review of data sources, methodology and comparability. $J$ Int AIDS Soc 2015;18:20634.

21. Haber N, Pillay D, Porter K, et al. Constructing the cascade of HIV care: methods for measurement. Curr Opin HIV AIDS 2016;11:102-8.

22. Heath K, Samji H, Nosyk B, et al. Cohort profile: Seek and treat for the optimal prevention of HIV/AIDS in British Columbia (STOP HIV/ AIDS BC). Int J Epidemiol 2014;43:1073-81.

23. Nosyk B, Montaner JSG, Colley G, et al. The cascade of HIV care in British Columbia, Canada, 1996-2011: a population-based retrospective cohort study. Lancet Infect Dis 2014;14:40-9.

24. Statistics Canada. Population by year, by province and territory (CANSIM table 051-0001. http://www.statcan.gc.ca/tables-tableaux/ sum-som/l01/cst01/demo02a-eng.htm (accessed 6 Dec 2017).

25. Public Health Agency of Canada. HIV and AIDS in Canada: Surveillance Report to December 31, 2014. https://www.canada.ca/ en/public-health/services/publications/diseases-conditions/hiv-aidscanada-surveillance-report-december-31-2014.html (accessed 27 Jan 2017).

26. Ontario HIV Epidemiology and Surveillance Initiative. New HIV diagnoses in Ontario. 2016 http://ohesi.ca/documents/OHESI-NewHIV-Diagnoses.pdf (accessed 27 Jan 2017).

27. Rourke SB, Gardner S, Burchell AN, et al. Cohort profile: the Ontario HIV Treatment Network Cohort Study (OCS). Int J Epidemiol 2013;42:402-11.

28. Burchell AN, Gardner S, Light L, et al. Implementation and Operational Research: Engagement in HIV Care Among Persons Enrolled in a Clinical HIV Cohort in Ontario, Canada, 2001-2011. J Acquir Immune Defic Syndr 2015;1999:e10-19.

29. Hashimoto RE, Brodt ED, Skelly AC, et al. Administrative database studies: goldmine or goose chase? Evid Based Spine Care $J$ 2014;5:74-6.
30. Hinds A, Lix LM, Smith M, et al. Quality of administrative health databases in Canada: a scoping review. Can J Public Health 2016;107:e56-61.

31. Jutte DP, Roos LL, Brownell MD. Administrative record linkage as a tool for public health research. Annu Rev Public Health 2011;32:91-108.

32. Cook JA, Collins GS. The rise of big clinical databases. Br J Surg 2015;102:e93-101.

33. Lundgren JD, Babiker AG, Gordin F, et al. INSIGHT START Study Group. Initiation of Antiretroviral Therapy in Early Asymptomatic HIV Infection. N Engl J Med 2015;373:795-807.

34. Wilton J, Liu J, Sullivan A, et al. Trends in HIV care cascade engagement among diagnosed people living with HIV in Ontario, Canada: A retrospective, population-based cohort study. PLoS One 2019;14:e0210096.

35. Ontario HIV Epidemiology and Surveillance Initiative. HIV testing in Ontario. 2016 http://ohesi.ca/documents/OHESI-HIV-testing-inOntario-in-2016.pdf (accessed 7 Feb 2019).

36. Lesko CR, Sampson LA, Miller WC, et al. Measuring the HIV care continuum using public health surveillance data in the United States. $J$ Acquir Immune Defic Syndr 2015;70:489-94.

37. Xia Q, Kersanske LS, Wiewel EW, et al. Proportions of patients with HIV retained in care and virally suppressed in New York City and the United States: higher than we thought. J Acquir Immune Defic Syndr 2015;68:351-8.

38. Wiewel EW, Braunstein SL, Xia Q, et al. Monitoring outcomes for newly diagnosed and prevalent HIV cases using a care continuum created with New York city surveillance data. J Acquir Immune Defic Syndr 2015;68:217-26.

39. MacCarthy S, Hoffmann M, Ferguson L, et al. The HIV care cascade: models, measures and moving forward. J Int AIDS Soc 2015;18:19395

40. Toren KG, Buskin SE, Dombrowski JC, et al. Time from HIV diagnosis to viral load suppression: 2007-2013. Sex Transm Dis 2016;43:34-40.

41. Harris TG, Rabkin M, El-Sadr WM. Achieving the fourth 90: healthy aging for people living with HIV. AIDS 2018;32:1563.

42. Autenrieth CS, Beck EJ, Stelzle D, et al. Global and regional trends of people living with HIV aged 50 and over: Estimates and projections for 2000-2020. PLoS One 2018;13:e0207005.

43. Ontario HIV Epidemiology and Surveillance Initiative 2017. HIV care cascade in Ontario: Linkage to care, in care, on antiretroviral treatment and virally suppressed. 2015 http://www.ohesi.ca/ documents/OHESI-cascade-report-17072017-a2.pdf (accessed Oct 2019).

44. Ontario HIV Epidemiology and Surveillance Initiative. HIV care cascade in Ontario by sex, age and health region: Linkage to care, in care, on antiretroviral treatment and virally suppressed. $2015 \mathrm{http}: / /$ ohesi.ca/documents/HIV-care-cascade-age-sex-health-region.pdf (accessed Oct 2019)

45. Granich R, Gupta S, Hall I, et al. Status and methodology of publicly available national HIV care continua and 90-90-90 targets: A systematic review. PLoS Med 2017;14:e1002253.

46. Porter K, Gourlay A, Attawell K, et al. Substantial heterogeneity in progress toward reaching the 90-90-90 HIV target in the $\mathrm{WHO}$ European Region. J Acquir Immune Defic Syndr 2018;79:28-37.

47. Antoniou T, Zagorski B, Loutfy MR, et al. Validation of case-finding algorithms derived from administrative data for identifying adults living with human immunodeficiency virus infection. PLoS One 2011;6:e21748.

48. Torian LV, Xia Q, Wiewel EW. Retention in care and viral suppression among persons living with HIV/AIDS in New York City, 2006-2010. Am J Public Health 2014;104:e24-9. 